NASA Technical Memorandum 88823

\title{
Optically Controlled GaAs \\ Dual-gate MESFET and \\ Permeable Base Transistors
}

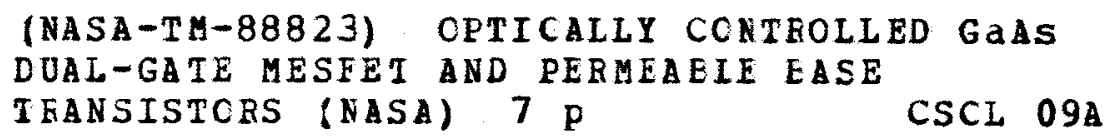

N $87-10232$

Unclas

G3/33 44160

R.N. Simons and K.B. Bhasin

Lewis Research Center

Cleveland, Ohio

Prepared for the

Conference on Lasers and Electro-Optics (CLEO)

cosponsored by the Institute of Electrical and Electronics Engineers

and the Optical Society of America

San Francisco, California, June 9-13, 1986 
OPTICALLY CONTROLLED GaAS DUAL-GATE MESFET AND PERMEABLE BASE TRANSISTORS

\author{
R.N. Simons* and K.B. Bhas in \\ National Aeronautics and Space Administration \\ Lewis Research Center \\ Cleveland, Ohio 44135
}

\begin{abstract}
SUMMARY
Direct optical control of microwave/millimeter wave devices can provide faster switching, gain and phase adjustability in amplifiers, and frequency tuning or locking in oscillators (ref. 1). Furthermore, it can reduce the complexity of fiber optic signal distribution networks for Gallium-Arsenide (GaAs) Monolithic Microwave Integrated Circuit (MMIC) based phased-array antennas for future space communication system.
\end{abstract}

Several authors have investigated the photovoltaic and photoconductive effect of light on the dc characteristics and the equivalent circult parameters of GaAs single-gate Metal Semiconductor Field Effect Transistors (MESFETs) (refs. 1 and 2). However, the single-gate MESFET has several 1imitations.

This paper presents computed results on light induced voltage, $v_{11 t}$, and its effect on the dc characteristics of both the GaAs dual-gate MESFET and the GaAs Permeable Base Transistor (PBT). The dual-gate MESFET when compared with the single gate MESFET has found a wide variety of applications. This is so because of the presence of two independent control gates together with exce1lent isolation characteristics. The PBT on the other hand has a much higher figure of merit, that is, the maximum frequency of oscillation. Typicaliy, the figure of merit of a PBT is $150 \mathrm{GHz}$ as compared to $60 \mathrm{GHz}$ for GaAs MESFET. Hence, PBTs can operate at a much higher speed than MESFETs.

Figure 1 is a schematic diagram illustrating the coupling of light from a laser to the active region, of a dual-gate MESFET and of a PBT, through an integrated optical waveguide and an optical fiber respectively. Light is responsible for the generation of excess electron-hole pairs in the active region; which in turn increases the minority carrier concentration, that is, holes in a n-type channel. An expression for the light induced voltage $v_{1 i t}$ as a function of the minority carrier concentration and other physical properties of GaAs is given in reference 3.

An expression relating drain current, $I_{d s}$, to the applied gate terminal voltages, $V_{g s} 1$ and $V_{g 2}$, and the drain to source voltage, $V_{d s}$, for $a$ depletion-mode (normaliy-ON) dual-gate MESFET is given in reference 4 . Illuminating either of the two gates of a dual-gate MESFET is equivalent to forward biasing that gate by a voltage source equal to vilt. The net voltage at that gate is therefore a superposition of the gate terminal voltage and vit. An expression relating drain current density $j$ to the applied gate bias $V_{g s}$, and $V_{d s}$ for a permeable-base transistor is given in reference 5 . The net voltage at the gate is a superposition of $V_{g s}$ and $v_{i i t}$.

\footnotetext{
*NRC-NASA Research Associate.
} 
The dual-gate MESFET active layer thickness, the PBT active layer thickness and their respective doping densities are indicated in figure 1. The intrinsic carrier concentration and the minority carrier life time are $1.79 \times 10^{6} \mathrm{~cm}^{3}$ and $10^{-8} \mathrm{sec}$ respectively. The wavelength of the incident light and the optical absorption coefficient in GaAs are normally 0.87 um and $10^{4} \mathrm{~cm}^{-1}$ respectively.

Using these equations light induced voltages are computed for the dualgate MESFET and the PBT as a function of the incident optical power density. This is shown in the inset in figure 2. The light induced voltage is observed to increase linearly with the incident optical power density. Besides, the two devices are almost equally sensitive to light.

The computed $I_{d s}$ of the dual-gate MESFET as a function of $V_{d s}$, with gate 2 optically 117 uminated, is shown in figure 2. Further, $I_{d s}$ increases by $16 \mathrm{~mA}$ with gate 1 opticaliy 11 luminated $\left(1 \mu \mathrm{W} / \mathrm{cm}^{2}\right)$ and $V_{\mathrm{gs}}=-1.5 \mathrm{~V}$ and $V_{\mathrm{g} 2}=0 \mathrm{~V}$. The computed drain current density $\mathrm{J}$ as a function of $V_{d s}$ for a PBT when the gate grating is 11 luminated is shown in figure 3. The dual gate MESFET provides additional control due to the presence of two independent control gates while the PBT can handle a much higher current density.

\section{REFERENCES}

1. R.G. Hunsperger, "Optical Control of Microwave Devices," in Integrated Optical Circuit Engineering II, SPIE, Vol. 578, S. Sriram, ed., Bellingham: SPIE, 1985, pp. 40-45.

2. A.A.A. DeSalles, "Optical Control of GaAs MESFET's," IEEE Trans. Microwave Theory Tech., vol. MTT-31,pp. 812-820, Oct. 1983.

3. G.J. Chaturvedi, R.K. Purohit and B.L. Sharma, "Optical Effect on GaAs MESFET's," Infrared Phys., vo1. 23, pp. 65-68, 1983.

4. R.A. Minasian, "Modelling DC Characteristics of Dual-Gate GaAs MESFET's," IEE Proc. Part I: Solid-State Electron Devices, vol. 130, pp. 182-186, Aug. 1983.

5. W.R.Frensley, "An Analytic Mode1 for the Barrier-Limited Mode of Operation of the Permeable Base Transistor," IEEE Trans. Electron Devices, vol. Ed-30, pp. 1624-1628, Dec. 1983. 


\section{ORIGISAL: PRGE is \\ OF POOR QUALITY}

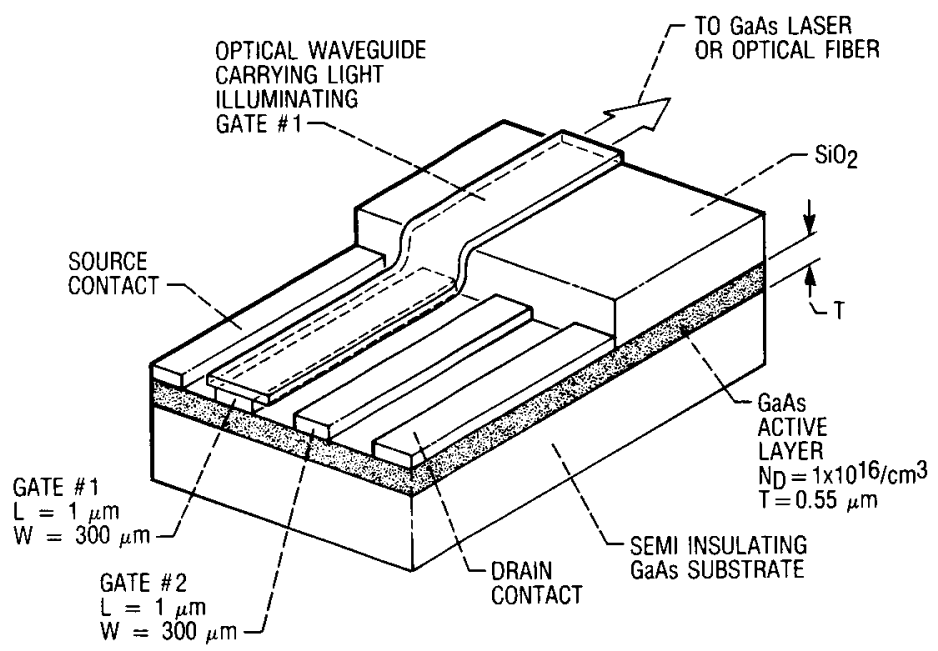

(a)

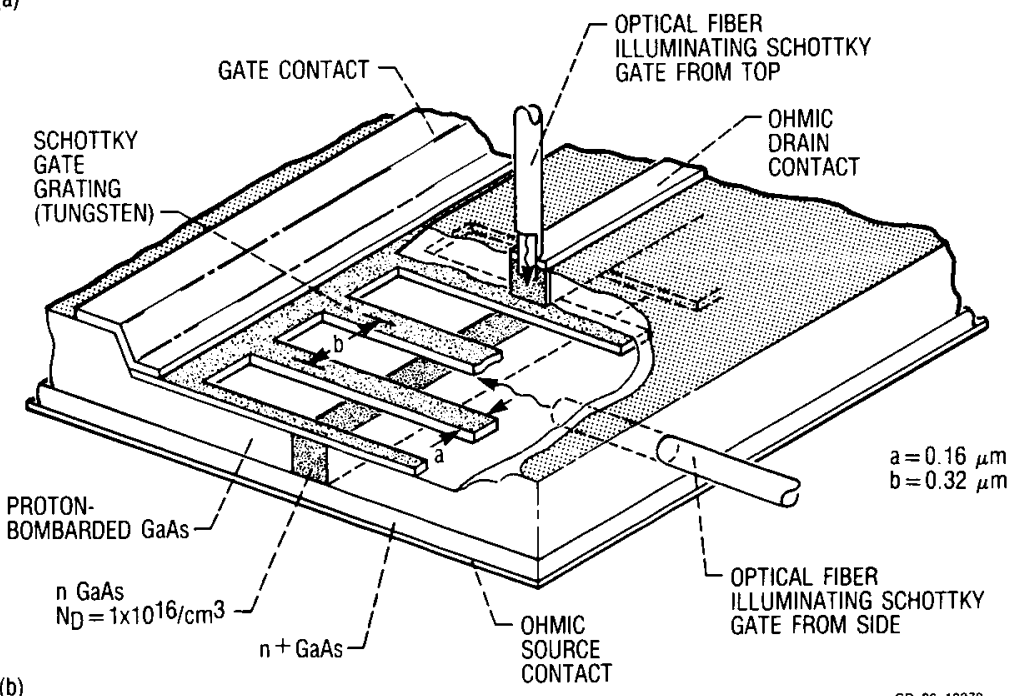

(b)

CD- $-86-18370$

Figure 1. - Schematic diagram illustrating (a) an optical waveguide illuminating a dual-gate MESFET. (b) an optical fiber illuminating a permeable base transistor at two possible locations. 


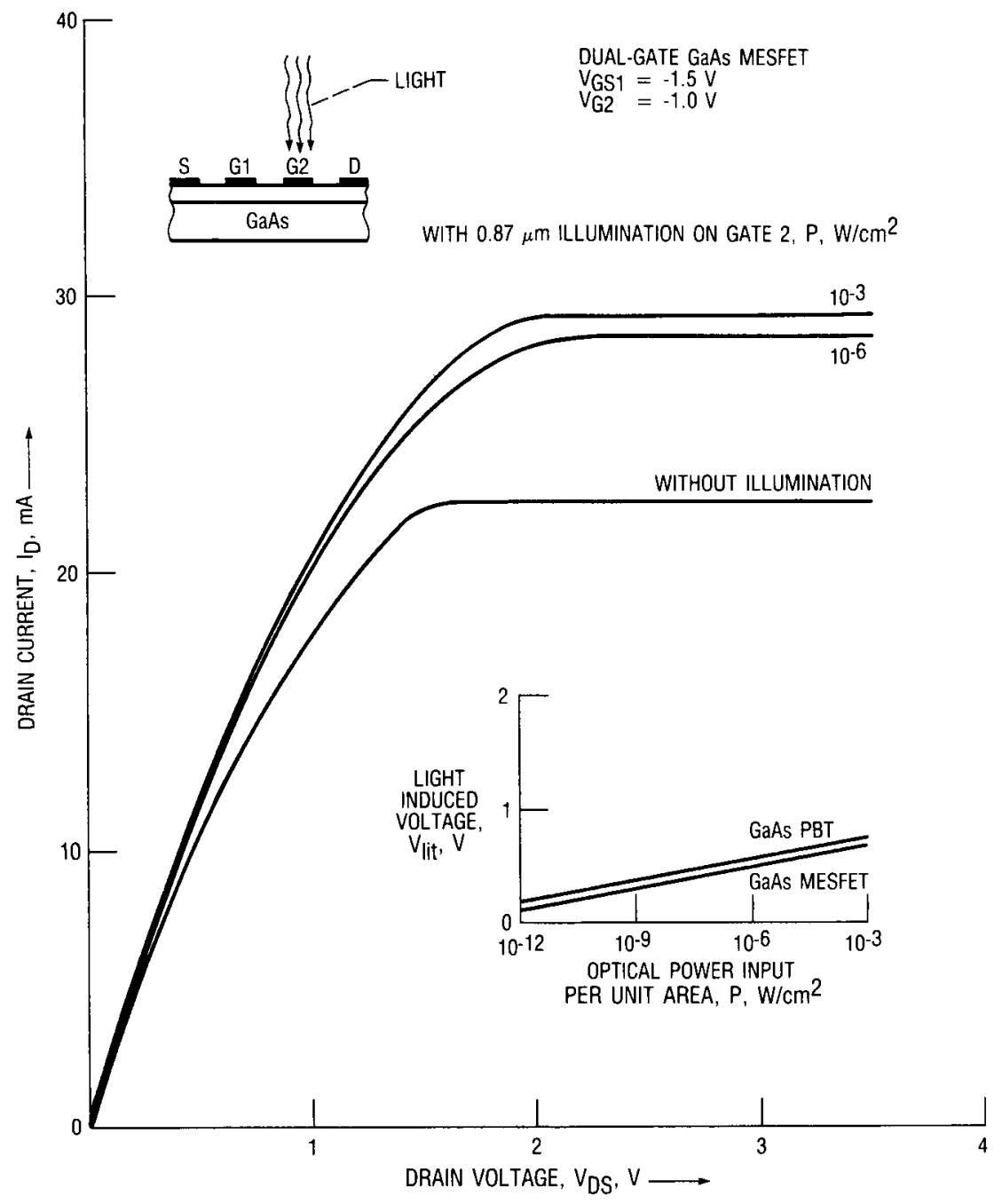

Figure 2 - Drain current versus drain to source voltage with gate 2 illuminated for a dualgate MESFET. Also shown in inset is the light induced voltage as a function of the incident optical power. 


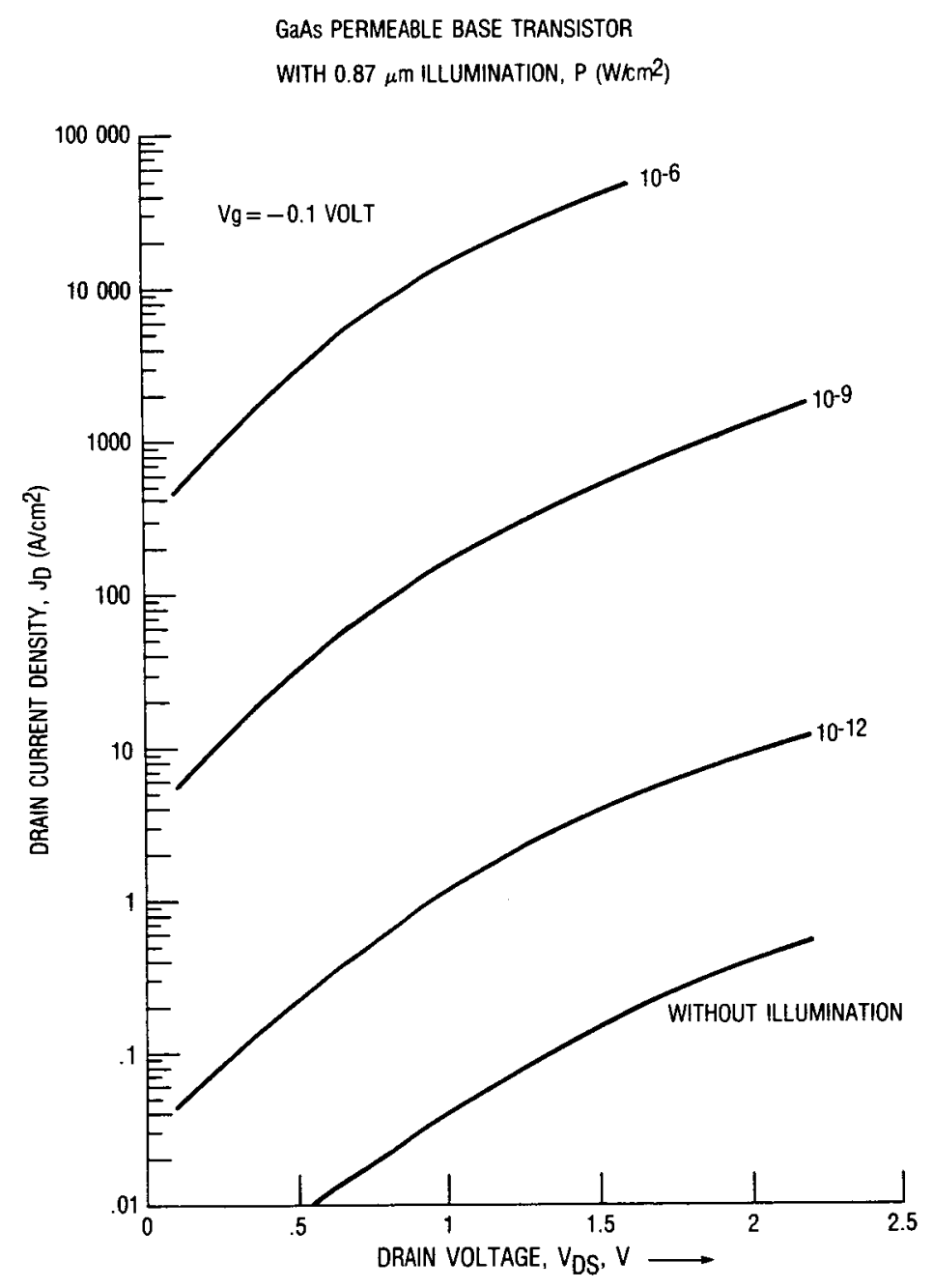

Figure 3. - Drain current density versus drain to source voltage with gate illumination for a permeable base transistor. 


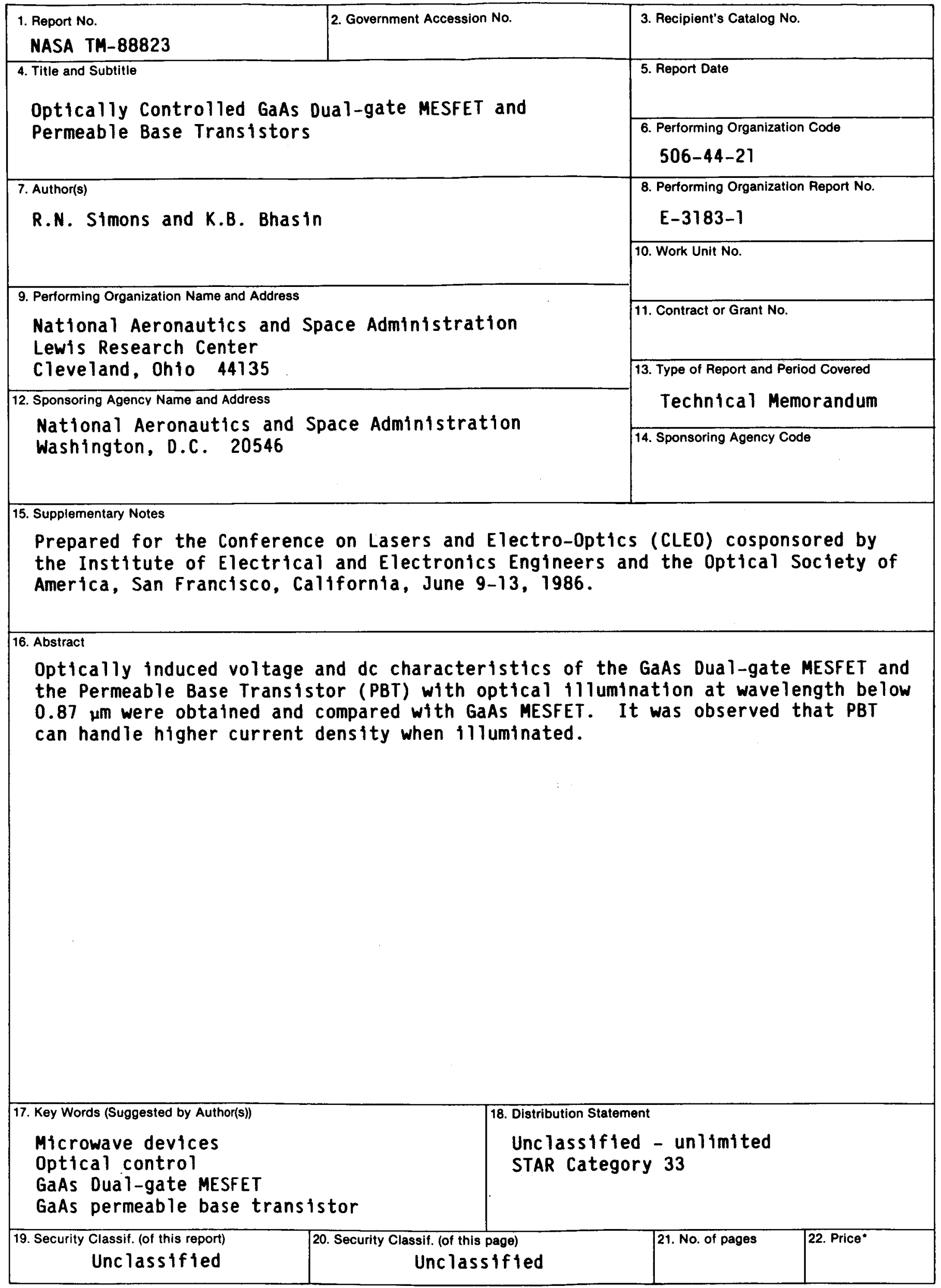

"For sale by the National Technical Information Service, Springfield, Virginia 22161 